\title{
007 SIMULATION OF HYDRAULICALLY FRACTURED WELLS
}

Y. DING' and E. CHAPUT ${ }^{2}$

'Institut Français du Pétrole, PO Box 311, 92506 Rueil-Malmaison, France

${ }^{2}$ Elf Aquitaine

\section{Introduction}

The technique of fracturing the wells is frequently used in petroleum engineering to improve the oil production. This technique is applied not only to vertical wells, but also to horizontal wells or multi-lateral wells. However, there are no efficient numerical methods to simulate these kinds of wells, especially for the fractured horizontal or multi-lateral wells. The handling of such a well by a reservoir simulator is particularly discussed in this paper.

The study of fractured wells has received more attention for a long time ${ }^{1-5}$. Some analytical or semi-analytical formulas have been developed, but these formulas cannot be used for the flow simulation with complex reservoir geometry and for a field study or for the study of the complex wells and fractures. To handle these cases, a reservoir simulator can be used.

During this decade, several numerical simulation studies for fractured wells have been published ${ }^{6-10}$. In all these studies, fine gridblocks are used around the wells and the fractures. Although using grid refinement for the fracture parallel to grid axes gives accurate simulation results, it is expensive and prohibitive for a full field simulation and there is no easy way to handle slanted fractures. So, it is necessary to study the techniques for fractured well simulations with coarse grid blocks.

In reservoir simulation, a numerical productivity index (PI) is usually introduced for the well modelling. In most of the well models ${ }^{11-15}$, the numerical PI is determined without considering the well trajectory and its position in the block. So these well models are inappropriate for the complex well and the fractured well modelling. Recently, a new kind of well model was proposed 16,17 , which takes into account the well trajectory by using equivalent transmissibilities to improve the flow calculation in the well vicinity. The applications of this well model to fractured wells are presented in this paper.

High conductivity fractures are particularly investigated, and these fractures can be considered as a part of the well. There are two kinds of fractures: 1). 2D fracture which is vertical and fully penetrates the reservoir thickness; 2). 3D fracture which is more general, and the fracture can be inclined and partially penetrated.

\section{Fractured Well Modelling}

The proposed well model is based on a steady-state pressure solution around the wells and the fractures. This pressure solution can be obtained using the boundary integral method ${ }^{17}$ as presented below.

\section{I Pressure solution for $2 D$ fracture}

For 2D problem, the pressure around the well and the fracture is given by:

$$
p(x)=-\frac{1}{2 \pi} \int_{\Gamma} \varphi(y) \ln (|x-y|) d \sigma_{y}
$$

where $\varphi(y)$, a density function defined at the wellbore and the fracture boundary $\Gamma$, satisfies the following boundary integral equation:

$$
p_{b c}(x)=-\frac{1}{2 \pi} \int_{\Gamma} \varphi(y) \ln (|x-y|) d \sigma_{y} \quad \forall x \in \Gamma
$$

with $\mathrm{pbc}(\mathrm{x})$ the pressure at $\Gamma$. Moreover, the normal pressure derivative at the wellbore boundary and the fracture boundary, which represents the production rate, can be calculated by:

$$
\left.\frac{\partial p(x)}{\partial n}\right|_{\Gamma}=-\frac{\varphi(x)}{2}-\frac{1}{2 \pi} \int_{\Gamma} \varphi(y) \frac{\partial}{\partial n_{x}} \ln (|x-y|) d \sigma_{y}
$$




\subsection{Pressure solution for $3 D$ fracture}

For 3D problem, the formulations are almost the same but with a different kernel. The pressure distribution around $3 \mathrm{D}$ fractures and wells is:

$$
p(x)=\frac{1}{4 \pi} \int_{\Gamma} \frac{\varphi(y)}{|x-y|} d \sigma_{y}
$$

where $\varphi(y)$ is the solution of the following boundary integral equation:

$$
p_{b c}(x)=\frac{I}{4 \pi} \int_{\Gamma} \frac{\varphi(y)}{|x-y|} d \sigma_{y} \quad \forall x \in \Gamma
$$

and the normal pressure derivative at the boundary $\Gamma$ is:

$$
\left.\frac{\partial p(x)}{\partial n}\right|_{\Gamma}=-\frac{\varphi(x)}{2}+\frac{1}{4 \pi} \int_{\Gamma} \varphi(y) \frac{\partial}{\partial n_{x}}\left(\frac{1}{|x-y|}\right) d \sigma_{y}
$$

\subsection{Well model}

Having the pressure distribution, the well model can be established by calculating the numerical PI and the equivalent transmissibilities around the wells and the fractures. As infinite conductivity fractures are considered as a part of the well, a numerical PI is also needed for each block containing the fractures. The numerical PI at a block is calculated by the following formula:

$$
P I_{b}=\frac{q_{b} \mu}{p_{0}-p_{w}}=k \int_{\Gamma_{b}} \frac{\partial p}{\partial \vec{n}} d \sigma /\left(p_{0}-p_{w}\right)
$$

where $q_{b}$ is the total well flow rate (including the flow rate in the fracture) in a grid block,

$\Gamma_{b}$ is the wellbore and the fracture boundary in a grid block,

$p_{w}$ is the average pressure at the wellbore and the fracture boundary in a block,

$p_{0}$ is the average pressure in a grid block containing wells or fractures.

The transmissibility between two blocks 1 and 2 in the well vicinity is calculated as:

$$
T_{12}=\frac{F_{12} \mu}{p_{I}-p_{2}}=k \int_{\Gamma_{12}} \frac{\partial p}{\partial \vec{n}} d \sigma /\left(p_{I}-p_{2}\right)
$$

where $F_{12}$ is the flow term through the interface $\Gamma_{12}, p_{1}$ and $p_{2}$ are the pressures at the blocks 1 and 2. If one of this block is a wellblock, the pressure equals to the average wellblock pressure.

As discussed in Ding ${ }^{17}$, the pressure solution corresponds always to some boundary conditions. Two kinds of boundary conditions are considered in our well model: 1). constant density condition $\varphi \equiv C$ which gives simple formula with analytical expression; and 2). infinite conductivity condition which corresponds to more realistic cases. The first case is also called uniform flux condition.

\section{Numerical Results}

The simulations of fractured well on coarse gridblocks are compared with semi-analytical solution 1,18 or fine grid simulations. In the fine grid simulation, the fractures are parallel to the grid axes and are located at the interface of the blocks as shown in Figure 1. The numerical productivity index (PI) is used at the blocks on both sides of the fracture. As the flow near the fracture is linear and perpendicular to the fracture, the numerical PI for a block $\mathrm{i}$ is calculated by the following formula:

$$
P I_{b}=k_{x} \Delta z_{i} \Delta y_{i} /\left(0.5 \Delta x_{i}\right)
$$

where $\Delta x_{i}, \Delta y_{i}$ and $\Delta z_{i}$ are the gridblock sizes, $k_{x}$ is the permeability in the $x$ direction. A similar approach was also used by Economides et $a l^{7}$ and Lefevre et $a l^{8}$. In coarse grid simulations, the new technique for well modelling presented in the previous section is applied. To get a comparison of coarse grid simulations, the results using linear PI by moving the fracture to the coarse gridblock interface are also presented.

In the following examples, the infinite conductivity fracture condition is assumed. A drawdown well test problem is simulated and the bottom hole pressures are checked. The simulation error, denoted as PI error, is evaluated by the following formula:

$$
\begin{aligned}
P I_{\text {err }} & =\left|\frac{\left(p_{w, \text { num }}-p_{a v}\right)-\left(p_{w, \text { ref }}-p_{a v}\right)}{p_{w, r e f}-p_{a v}}\right| \times 100 \% \\
& =\left|\frac{p_{w, \text { num }}-p_{w, r e f}}{p_{w, \text { ref }}-p_{a v}}\right| \times 100 \%
\end{aligned}
$$


where $p_{w, n u m}$ is the wellbore pressure obtained by the coarse grid simulation, $p_{w, \text { ref }}$ is the reference wellbore pressure, and $p_{a v}$ is an average reservoir pressure. This error will be estimated for a time long enough so that the steady-state or pseudo steady-state flow is reached in the vicinity of wells and fractures. It represents the error for the well performance calculation.

\section{Example 1. Hydraulically fractured vertical well}

A vertical fracture of $100 \mathrm{~m}$ penetrates fully the reservoir thickness. This is a $2 \mathrm{D}$ problem. Coarse gridblocks with size of $100 \mathrm{~m}$ are used in the simulation (Figure 2). It has to be mentioned that it is inconvenient using Peaceman's well model for the coarse grid simulation. Peaceman's well model gives a negative numerical PI, because the equivalent wellblock radius $r_{0}=20 \mathrm{~m}$ is smaller than the equivalent wellbore radius $r_{w, e q}=x_{\mathrm{f}} / 2=25 \mathrm{~m}$. Using the linear PI (Eq. (19)) by placing the well at the grid interface is possible, but the error is important. The fine grid discretization around the fracture is given in Table 1, where the fracture blocks are underlined with bold characters. Figure 3 represents the comparison results for semi-analytical model, fine grid simulation and coarse grid simulation using the linear PI or using the new well model with the uniform flux condition or with the infinite conductivity condition. The PI error is checked at $100 \mathrm{~h}$, when the flow is stabilised. If the semi-analytical solution is considered as the reference solution, the error of fine grid simulation is $0.8 \%$ and the error for coarse grid simulation is $6.1 \%$ when using the uniform flux condition for the new well model and $0.7 \%$ when using the infinite conductivity condition in the model (Figure 4). While the error caused by using linear PI is $39 \%$.

For coarse grid simulation, using the new well model with the infinite conductivity condition gives satisfied result. Using the uniform flux condition is less accurate, but the result is much better than that using the linear PI. The fine grid simulation gives a reasonable accurate results with a error less than $1 \%$. So, in more complex cases as shown in the following examples, the fine grid simulations are considered as the reference solution.

\section{Example 2. Multi-fractured horizontal well}

A horizontal well is fractured at several locations along the horizontal section. The fracture height is the entire reservoir thickness. Assuming that the formation fluid cannot flow directly into the wellbore and the only contribution for the well production is from the fractures ${ }^{3}$, this problem can be treated again as a $2 \mathrm{D}$ problem. The simulations for 1 fracture, 3 fractures and 5 fractures are considered (Figure 5). The fracture half length is $150 \mathrm{~m}$ and the distance between fractures is $200 \mathrm{~m}$. The coarse gridblock size is of $100 \mathrm{~m}$. The comparison of coarse grid with fine grid simulations are given in Figure 6. When simulated with linear PI, the grid blocks are displaced so that the fractures are at the interfaces. It is clear that using the new well model gives always better results, and the new well model with the infinite conductivity condition gives very satisfactory results with errors of order of $1 \%$.

\section{Example 3. Non fully penetrated fracture}

This example shows the simulations for the fracture not fully penetrating the reservoir thickness. For these simulations, the 3D pressure solution is used for the new well model.

An horizontal well of $500 \mathrm{~m}$ is intersected by a vertical fracture of length $300 \mathrm{~m}$ and height $30 \mathrm{~m}$ in a slab reservoir. The reservoir thickness is $90 \mathrm{~m}$ and the fracture is located at the middle of the reservoir as shown in Figure 7 . The vertical permeability and horizontal permeability ratio is 0.1 . The coarse grid sizes are of $100 \mathrm{~m} \times 100 \mathrm{~m} \times 10 \mathrm{~m}$. Two cases are considered in this example (Figure 8):

\section{- Case 1). production only from the fracture}

The new well model is robust. It is not necessary that the fracture parallels to the grid axes. Two situations are considered in coarse grid simulation with the fracture parallel to $y$ direction (Case 1a) or slanted with $45^{\circ}$ (Case $1 \mathrm{~b}$ ). The fine grid simulation is used only for the Case $1 \mathrm{a}$, where the fracture is parallel to grid axes. This fine grid simulation gives also the reference solution for the Case $1 b$, because the reservoir is infinite in the horizontal plane. Figure 9a represents the coarse grid simulation errors. For the coarse grid simulation, the linear PI can only be reasonably used for the Case 1a.

- Case 2). production from the fracture as well as from the well

To obtain a reliable reference solution by the fine grid simulation, the fracture is assumed to be perpendicular to the well. Again, two situations are considered in coarse grid simulation with the fracture parallel to $y$ direction (Case $2 \mathrm{a}$ ) or slanted with $45^{\circ}$ (Case $2 \mathrm{~b}$ ). The fine grid simulation is used only for the Case 2a. For the fine grid simulation, linear PI is used for the fracture and Peaceman's PI is used for the well. Figure $9 \mathrm{~b}$ represents the coarse grid simulation errors.

In all the two cases, using the new well model gives better results, especially for the new well model with the infinite conductivity condition. This example shows the ability using the new well model to simulate complex fractures such as non fully penetrated fractures or slanted fractures. 


\section{Conclusions}

The simulation of fractured wells by a reservoir simulator is discussed. Using grid refinement around the wells and the fractures can give satisfactory results, but it is expensive and prohibitive for full field studies.

In this paper, the methodology for the simulation of fractured wells on coarse gridblocks is discussed, and a new technique is applied. Some applications are presented for infinite conductivity fractured wells, and the advantages of the new method are shown. In all the examples, the results of coarse grid simulation using the new method is much better than those using the linear PI. Using the infinite conductivity for the new well model gives the results very close to those of fine grid simulation. The new method can be used to handle fully penetrated fractures as well as non fully penetrated fractures. It can also be used to handle slanted fractures, which are hardly managed using grid refinement.

\section{Acknowledgements}

The authors would like to thank ARTEP for the support to this work.

\section{References:}

1. Gringarten, A.C., Ramey, H.J., and Raghavan, R.: "Pressure Analysis for Fractured Wells," paper SPE 4051, San Antonio, Oct. 1972.

2. Cinco-Ley, H. and Samaniego, F.: "Transient Pressure Analysis for Fractured Wells," JPT (Spet. 1981) 1749.

3. Soliman, M.Y., Hunt, J.L. and El Rabaa, A.M.: "Fracturing Aspects of Horizontal Wells," JPT (Aug. 1990) 966

4. Raghavan, R. Chen, C. and Agarwal, B.: "An Analysis of Horizontal Wells Intercepted by Multiple Fractured," paper SPE 27652 presented at the Permian Basin Oil and Gas Rec. Conf. Midland, TX. 16-18 March 1994.

5. Larsen, L. and Hegre, T.M.: "Pressure Transient Analysis of Multifractured Horizontal Wells," paper SPE 28389 presented at the SPE 69th ATC\&E, New Orleans, Spet. 1994.

6. Conlin, J.M., Hale, J.L., Sabathier, J.C., Faure, F. and Mas, D.: "Multiple-Fracture Horizontal Wells: Performance and Numerical Simulation," paper SPE 20960 presented at Europec, the Hague, Netherlands, 22-24, Oct. 1990.

7. Economides, M.J., Deimbacher, F.X., Brand, C.W., and Heinemann, Z.E.,: "Comprehensive Simulation of Horizontal-Well Performance," SPEFE (Dec. 1991) 418.

8. Lefevre, D., Pellissier, G. and Sabathier, J.C.: "A New Reservoir Simulation System for a Better Reservoir Management," paper SPE 25604 presented at the SPE Middle East Oil Tech. Conf. \& Exhibition, Bahrain, 3-6 April 1993.

9. Hegre, T.M.: "Hydraulically Fractured Horizontal Well Simulation," paper SPE presented at the European 3-D Reservoir Modelling Conference, Stavanger, Norway, 16-17 April 1996.

10. Kroemer, E., Abou-Sayed, I.S., Babu, D.K. and Cohen, M.F.: "Compositional Simulation of Well Performance for Fractured and Multiple Fractured Horizontal Wells in Stratified Gas Condensate Reservoirs," paper SPE 37995 presented at the SPE Reservoir Simulation Symposium, Dallas, 8-11 June 1997

11. Peaceman, D.W.: "Interpretation of Wellblock Pressure in Numerical Reservoir Simulation Part 3--Off Center and Multiple Wells Within a Wellblock," paper SPE 16976 presented at the 1987 SPE ATC\&E, Dallas, Sept. 27-30.

12. Babu, D.K., Odeh, A.S., Al-Khalifa, A.J. and McCann, R.C.: "The Relation Between Wellblock and Wellbore Pressures in Numerical Simulation of Horizontal Wells," SPERE (Aug. 1991) 324-328.

13. Peaceman, D.W.: "Representation of a Horizontal Well in Numerical Reservoir Simulation," SPE Adv. Technology Series, Vol 1, No 1 (1993) 7-16.

14. Chen, G., Tehrani, D.H. and Peden, J.M.: "Calculation of Well Productivity in a Reservoir Simulator (I)", paper SPE 29121 presented at the 13th SPE Symposium on Reservoir Simulation, San Antonio, TX, Feb. 12-15, 1995.

15. Mochizuki, S.: "Well Productivity for Arbitrarily Inclined Well", paper SPE 29133 presented at the 13th SPE Symposium on Reservoir Simulation, San Antonio TX Feb. 12-15, 1995.

16. Ding, Y. and Renard, G.: "A New Representation of Wells in Numerical Reservair Simulation," SPERE (May 1994) 140.

17. Ding, Y: "A Generalized 3D Well Model for Reservoir Simulation," SPEJ (Dec. 1996) 437.

18. Besson, J.: "Performance of Slanted and Horizontal Wells on an Anisotropic Medium," paper SPE 20965 presented at Europec, Hague, Netherlands, 22-24 Oct. 1990.

\section{Table 1. Grid discretisation around the fracture}

\begin{tabular}{|c|c|}
\hline$\Delta \mathrm{x}(\mathrm{m})$ & $\Delta y(m)$ \\
\hline $\begin{array}{l}3(100) ; 9(40) ; 20(20) ; 2(10) ; 2(5) ; 3(2.5) ; 4(0.5) ; \\
0.5 ; 0.5 ; 4(0.5) ; 3(2.5) ; 2(5) ; 2(10) ; 20(20) ; \\
9(40) ; 3(100)\end{array}$ & $\begin{array}{l}100 ; 9(40) ; 20(20) ; 5(10) ; 2(2.5) ; 4(0.625) ; \\
13(2.5) ; 6(5) ; 13(2.5) ; 4(0.625) ; 2(2.5) ; \\
9(5) ; 5(10) ; 20(20) ; 9(40) ; 100\end{array}$ \\
\hline
\end{tabular}




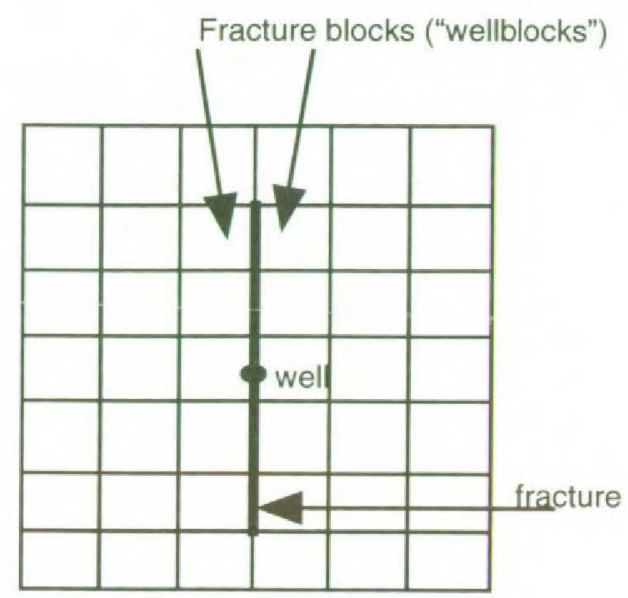

Figure 1--Fractured well modelling with the linear PI formula Eq.(9)

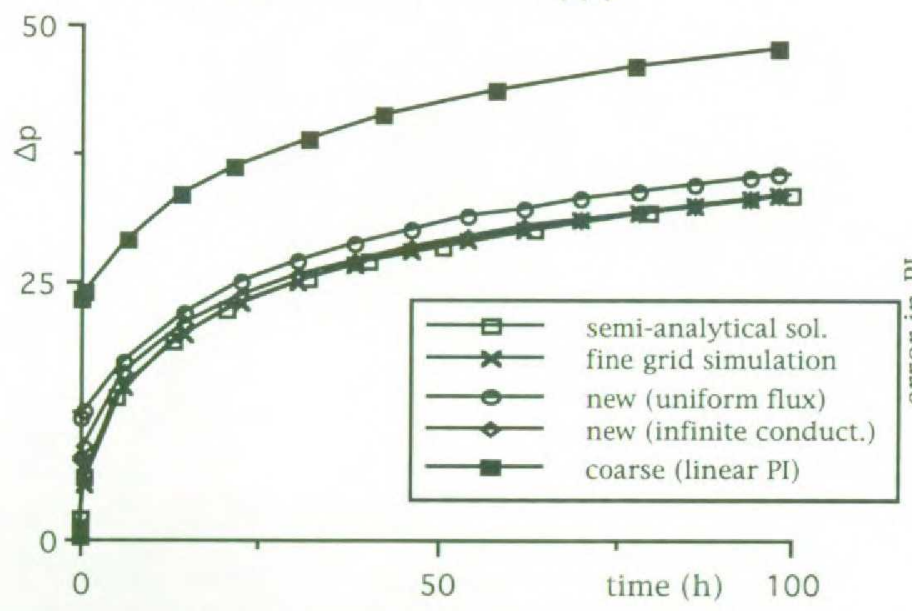

Figure 3-- Wellbore pressure for hydraulically fractured vertical well

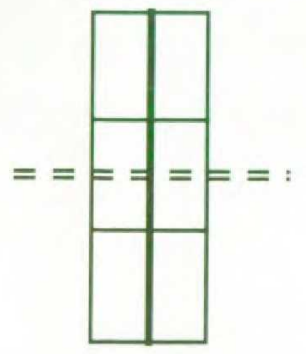

5a). One fracture

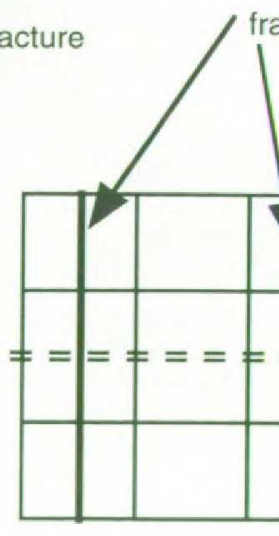

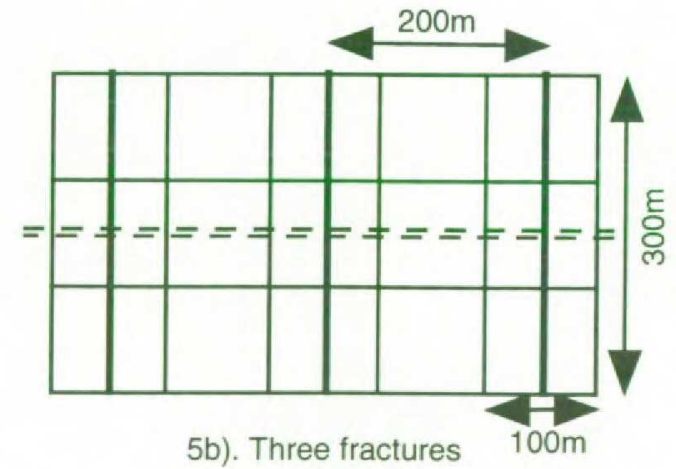

$5 b)$. Three fractures

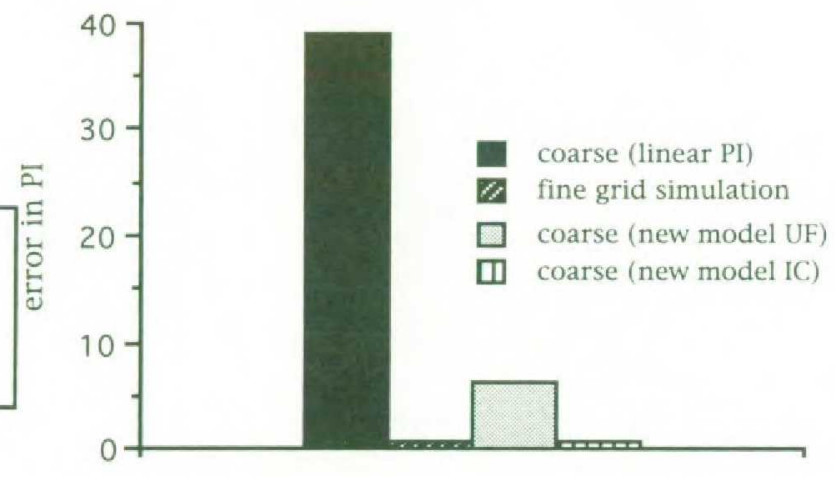

Figure 4--PI error at 100 hours in example 1
Figure 2--Coarse grid simulation for hydraulically fractured vertical well 


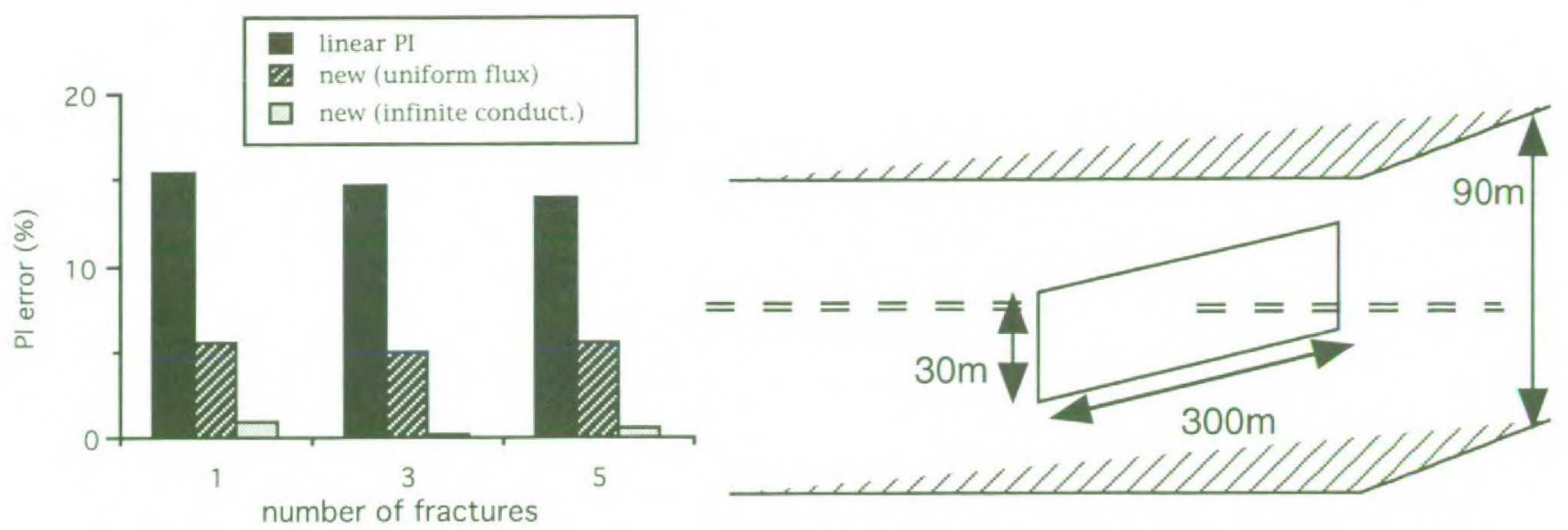

Figure 6--Coarse grid simulation errors for multi-fractured well

Figure 7--Non fully penetrated fracture in a slab reservoir

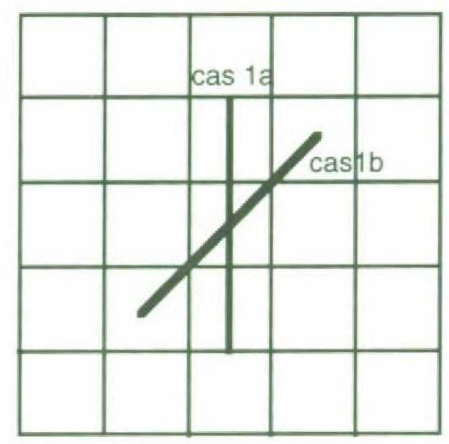

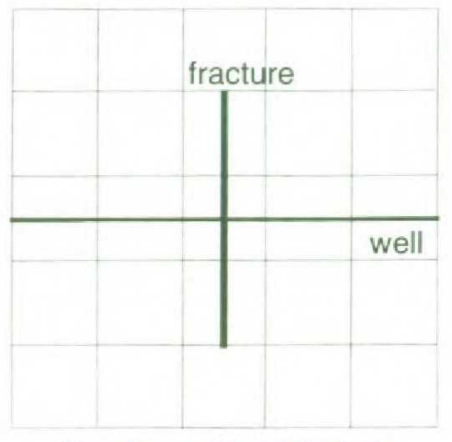

Case $2 \mathrm{a}-$ well and fracture parallel to grid axes

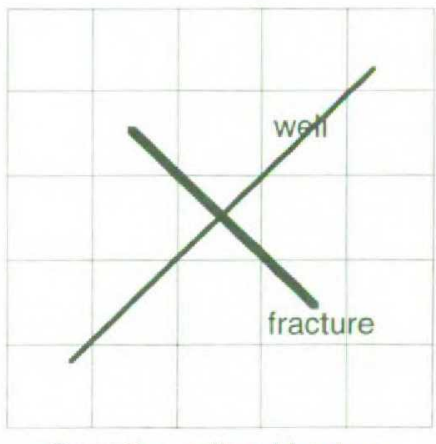

Case $2 \mathrm{~b}$ - well and fracture non parallel to grid axes

Case 1 - production only from the fracture Case 2 - flow production from the fracture as well as from the well

Figure 8--Coarse grid simulation (in xy plane) for non fully penetrated frectured well

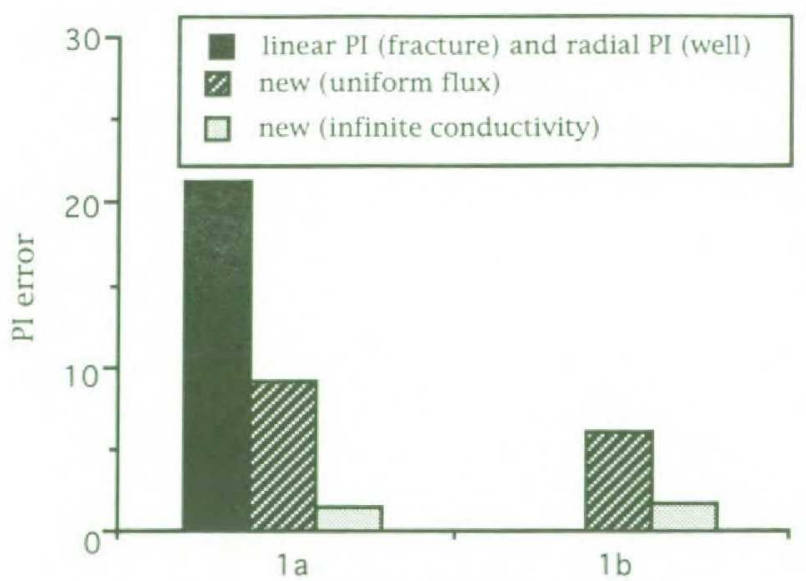

9a). Errors in Case 1

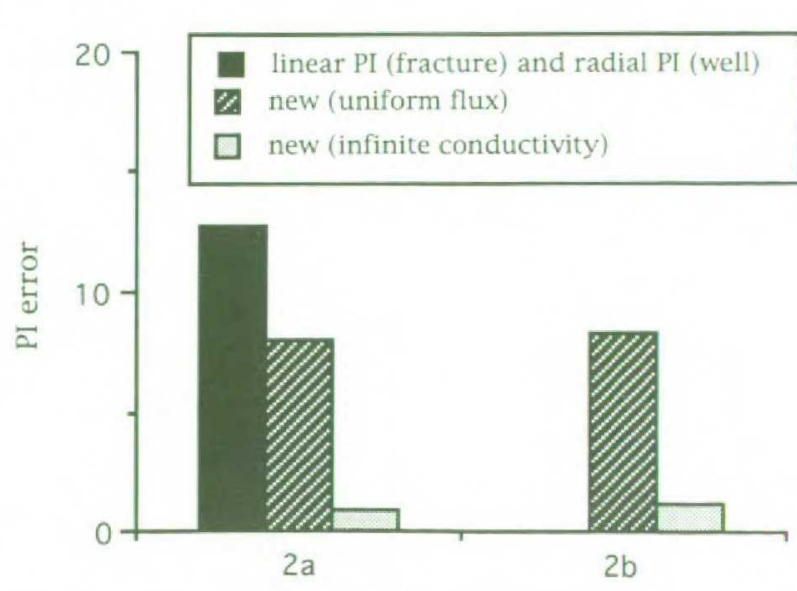

$9 b)$. Errors in Case 2

Figure 9--Simulation error for non fully penetrated fracture 